\title{
Universal Systems for Entropy Intervals
}

\author{
Tomasz Downarowicz $^{1,2}$ • Jacek Serafin ${ }^{1}$
}

Received: 28 October 2015 / Published online: 1 February 2016

(C) The Author(s) 2016. This article is published with open access at Springerlink.com

\begin{abstract}
A topological system is universal for a class of ergodic measure-theoretic systems if its simplex of invariant measures contains, up to an isomorphism, all elements of this class and no elements from outside the class. We construct universal systems for classes given by the combination of three properties: measure-theoretic entropy belonging to a nondegenerate interval of the extended nonnegative real halfline, invertibility and aperiodicity. For classes consisting of aperiodic systems the universal system can be made minimal.
\end{abstract}

Keywords Ergodic automorphism · Ergodic endomorphism · Entropy · Topological dynamical system · Universal system

Mathematics Subject Classification 37B05 $\cdot 37$ A05 $\cdot 37$ A35 $\cdot 37$ B10

\section{Description of the Result}

We will consider topological dynamical systems $(Z, F)$ where $Z$ is a compact metric space and $F: Z \rightarrow Z$ is a homeomorphism or just a continuous map.

Classically, a system $(Z, F)$, with $F$ being a homeomorphism, is called universal if for every ergodic system $(\mathcal{X}, \mathcal{F}, \mu, T)$, where $T: \mathcal{X} \rightarrow \mathcal{X}$ is an automorphism of a standard nonatomic probability space $(\mathcal{X}, \mathcal{F}, \mu)$ whose Kolmogorov-Sinai entropy $h_{\mu}(T)$ is strictly smaller than the topological entropy $h_{\text {top }}(Z, F)$, there exists an $F$-invariant measure $v$ on $Z$ such that the systems $(\mathcal{X}, \mathcal{F}, \mu, T)$ and $\left(Z, \mathcal{B}_{v}, v, F\right)$, with $\mathcal{B}_{v}$ denoting the Borel sigma-

Tomasz Downarowicz

downar@pwr.edu.pl

Jacek Serafin

serafin@pwr.edu.pl

1 Faculty of Pure and Applied Mathematics, Wrocław University of Technology, Wybrzeże Wyspiańskiego 27, 50-370 Wrocław, Poland

2 Institute of Mathematics, Polish Academy of Sciences, Śniadeckich 8, 00-656 Warsaw, Poland 
algebra in $Z$ completed with respect to $v$, are measure-theoretically isomorphic. We will shortly pronounce the last condition as $(Z, F)$ supports a measure isomorphic to $\mu$. Notice that so understood universality is insensitive to whether the system has periodic points or carries measures of maximal entropy. Krieger Generator Theorem [6] is equivalent to saying that the full shift is universal. The notion has been studied in various contexts, in particular for toral automorphisms [7] or systems with specification [8].

We will adopt the following, slightly stronger and more precise, notion of universality: a system $(Z, F)$ is said to be universal for a class $\mathcal{H}$ of ergodic endomorphisms (i.e., not necessarily invertible measure-theoretic dynamical systems understood modulo isomorphism) whenever three conditions hold:

(1) For every system $(\mathcal{X}, \mathcal{F}, \mu, T)$, where $T: \mathcal{X} \rightarrow \mathcal{X}$ is an ergodic endomorphism of a standard probability space $(\mathcal{X}, \mathcal{F}, \mu)$, belonging to the class $\mathcal{H},(Z, F)$ supports a measure isomorphic to $\mu$.

(2) Conversely, for every ergodic $F$-invariant Borel probability measure $v$ on $Z$, the system $\left(Z, \mathcal{B}_{v}, v, F\right)$ belongs to $\mathcal{H}$.

(3) If $\mathcal{H}$ consists exclusively of automorphisms, a universal system should be invertible ( $F$ should be a homeomorphism).

We are interested in the existence of universal systems for classes determined by inequalities imposed upon the Kolmogorov-Sinai entropy, invertibility and aperiodicity. And so, for an interval $I \subset[0, \infty]$ (which can be closed or open on either side, and may but need not contain infinity), we define $\mathcal{H}_{I}^{e}$ as the class of all ergodic endomorphisms whose KolmogorovSinai entropy is contained in the interval $I$. Also, we define $\mathcal{H}_{I}^{\mathrm{a}}$ as the class of all ergodic automorphisms contained in $\mathcal{H}_{I}^{\mathrm{e}}$. Additionally, if $0 \in I$ we will distinguish the classes $\tilde{\mathcal{H}}_{I}^{\mathrm{e}}$ and $\tilde{\mathcal{H}}_{I}^{\text {a }}$ consisting of aperiodic elements of $\mathcal{H}_{I}^{\mathrm{e}}$ and $\mathcal{H}_{I}^{\mathrm{a}}$, respectively (if $0 \notin I$, aperiodicity is obvious). The question is: which of the above classes possess a universal system, and which do not.

Universal systems for the classes $\mathcal{H}_{[0, \infty]}^{\mathrm{a}}$ (of all automorphisms) and $\mathcal{H}_{[0, \infty]}^{\mathrm{e}}$ (of all endomorphisms) are well known to exist— the full shift (bilateral or unilateral, respectively) with a Cantor alphabet is an obvious example. While universal systems for $\mathcal{H}_{[0, C)}^{\mathrm{a}}$ and $\mathcal{H}_{[0, C)}^{\mathrm{e}}$ (for any $0<C \leq \infty$ ) can be easily created as countable unions of universal systems in the classical sense, the question for other types of entropy intervals remained, until recently, completely open. For closed entropy intervals $[0, C]$, the requirement (2) makes the definition of universality much more demanding than the one considered earlier. In a recent paper of the second author [10] a somewhat surprising result has been proved: $\mathcal{H}_{\{0\}}^{\mathrm{a}}$ does not admit a universal system (and an analogous result can be deduced for $\mathcal{H}_{\{0\}}^{\mathrm{e}}$ ). We are not aware of any previous results concerning intervals not containing 0 . In this paper we will fill in the missing cases by showing that in most situations the universal systems do exist. We leave open the cases of degenerate (one-point) intervals $\{c\}$ where $c \neq 0$. For technical reasons we also leave out the noninvertible cases where $I$ is bounded and does not contain 0 , but we strongly believe that the universal systems can be created by the same methods as used for automorphisms once the necessary modifications are made in some of the classical results of Jewett-Krieger type.

Let us also mention results combining the notion of universality with minimality. Weiss constructed a minimal system universal in the class of all aperiodic automorphisms [12]. The first author of this paper extended this result in [1] to aperiodic endomorphisms (also obtaining a minimal universal system). There are also results by Shilon and Weiss [11] presenting minimal bilateral subshifts universal (in the classical sense) for the class of aperiodic automorphisms with entropy in $[0, C)$, however these systems admit (inevitably, as they are 
subshifts) some exceptional measures with maximal entropy $C$. Since these measures do not represent all ergodic systems with this entropy value, their examples are not universal (for neither $\tilde{\mathcal{H}}_{[0, C)}^{\mathrm{a}}$ nor $\tilde{\mathcal{H}}_{[0, C]}^{\mathrm{a}}$ ) in our stronger understanding. We will address the possibility of creating minimal universal systems for the classes $\tilde{\mathcal{H}}_{I}^{\mathrm{a}}$ and $\tilde{\mathcal{H}}_{I}^{\mathrm{e}}$ in Sect. 6 (strengthening the results of [11]).

\section{Topological Inverse Limits of Subshifts}

Throughout this paper by a subshift we will mean any closed subset of a symbolic space $\Lambda^{\mathbb{S}}\left(\Lambda\right.$ is a finite set called alphabet and $\mathbb{S}=\mathbb{Z}$ or $\mathbb{S}=\mathbb{N}_{0}=\{0,1,2, \ldots\}$, depending on whether we discuss invertible or noninvertible case), invariant under the standard shift map $(S x)(i)=x(i+1)\left(x \in \Lambda^{\mathbb{S}}\right)$. Suppose we have a sequence of subshifts $\left(X_{k}, S_{k}\right)(k \geq 1$, each possibly on a different alphabet $\left.\Lambda_{k}\right)$ such that for each $k,\left(X_{k}, S_{k}\right)$ is a topological factor of $\left(X_{k+1}, S_{k+1}\right)$. Let $\pi_{k}: X_{k+1} \rightarrow X_{k}$ be the corresponding factor map (recall that such map is always a block code with finite horizon).

Definition 2.1 The inverse limit

$$
(X, T)=\overleftarrow{\lim _{k \rightarrow \infty}}\left(X_{k}, S_{k}\right)
$$

is defined as the set

$$
X=\left\{\left(x_{k}\right)_{k \geq 1} \in \prod_{k \geq 1} X_{k}: x_{k}=\pi_{k}\left(x_{k+1}\right) \text { for all } k \geq 1\right\},
$$

with $T$ acting coordinatewise by the shifts $S_{k}$.

The set $X$ defined above is zero-dimensional, closed and $T$-invariant, hence $(X, T)$ is a zerodimensional topological dynamical system. Conversely, every zero-dimensional topological dynamical system $(X, T)$ is topologically conjugate to an inverse limit of unilateral subshifts (also bilateral, if $T$ is invertible). Indeed. First notice that every clopen partition $\mathcal{P}$ of $X$ determines a symbolic system formed by the (unilateral or bilateral, depending on the meaning of $\mathbb{S}) \mathcal{P}$-names of the points $x \in X$ and it is easy to see that this subshift is a topological factor of $(X, T)$. We will call this factor the $\mathcal{P}$-process. Now, let $\mathcal{P}_{1}, \mathcal{P}_{2}, \ldots$ be a sequence of clopen partitions of $X$ such that $\mathcal{P}_{k+1} \succcurlyeq \mathcal{P}_{k}$ for each $k \geq 1$ and $\operatorname{diam}\left(\mathcal{P}_{k}\right) \rightarrow 0$, and let $\left(X_{k}, S_{k}\right)$ denote the $\mathcal{P}_{k}$-process. It is easy to see that for each $k \geq 1,\left(X_{k}, S_{k}\right)$ is a topological factor of $\left(X_{k+1}, S_{k+1}\right)$ (via a one-block code, i.e., by a translation of single symbols). Since the partitions $\mathcal{P}_{k}$ jointly separate points, it is clear that the corresponding inverse limit is topologically conjugate to $(X, T)$.

Any zero-dimensional system $(X, T)$, as an inverse limit of subshifts $\left(X_{k}, S_{k}\right)$, has a convenient array representation, in which the elements of $X$ are two-dimensional arrays $x=[x(k, i)]_{k \geq 1, i \in \mathbb{S}}$ consisting of countably many rows, where the $k$ th row $x_{k}$ is an infinite (unilateral or bilateral) sequence over some finite alphabet $\Lambda_{k}$. The rule behind the assignment is

$$
x(k, i)=\lambda \in \Lambda_{k} \Longleftrightarrow T^{i}(x) \in P_{\lambda} \in \mathcal{P}_{k},
$$

where $\lambda \mapsto P_{\lambda}$ is a bijective labeling of $\mathcal{P}_{k}$ by $\Lambda_{k}$ (in other words, the $k$ th row is the $\mathcal{P}_{k}$-name of $x$ ). The map $T$ acts on the arrays as the horizontal shift $(T x)(k, i)=x(k, i+1)$ for all 
$k, i$. From now on we will use the letter $S$ (or $S_{k}$ ) to denote the shift in symbolic systems, while $T$ (or $T_{k}, T^{\prime}$, etc.) will be used for more general transformations including shifts on arrays.

We will exploit an inverse limit representation with special properties. ${ }^{1}$ We formulate it for automorphisms. The noninvertible case will be discussed separately, see Remark 2.6.

Theorem 2.2 Fixan interval $I=[c, C](0 \leq c<C \leq \infty)$. Let $\left(X_{1}, S_{1}\right)$ be a strictlyergodic bilateral subshift which is a Jewett-Krieger model for the Bernoulli shift with entropy $c$ (if $c=0$ then we let $\left(X_{1}, S_{1}\right)$ be the trivial subshift over one symbol). Then, for every ergodic automorphism $(\mathcal{X}, \mathcal{F}, \mu, T)$ with entropy $h \in I$, there exists an increasing sequence of strictly ergodic subshifts $\left(X_{1}, S_{1}\right) \leftarrow\left(X_{2}, S_{2}\right) \leftarrow \cdots$ (the first one being the selected Bernoulli model), each having entropy strictly smaller than $C$, and such that the inverse limit

$$
(X, T)=\overleftarrow{\lim _{k \rightarrow \infty}}\left(X_{k}, S_{k}\right)
$$

is a strictly ergodic model for $(\mathcal{X}, \mathcal{F}, \mu, T)$.

The proof relies on the following construction, which we call a mix of two processes (in the definition and the following lemma we do not need to assume invertibility of $T$ ):

Definition 2.3 Let $(\mathcal{X}, \mathcal{F}, \mu, T)$ be a measure-theoretic dynamical system (endomorphism or automorphism). Let $\mathcal{P}$ and $\mathcal{Q}$ be two finite measurable partitions of $\mathcal{X}$. Fix a coefficient $0<p<1$. Choose a Rokhlin tower of height $n$ and remainder of measure at most $\frac{1}{n}$ (we will say an " $n$-tower"). Denote by $\mathcal{W}$ the partition into two sets: the union $P$ of the lower $\lfloor p n\rfloor$ levels of the tower and its complement $Q$. Let $\mathcal{R}=\operatorname{MIX}_{n}(p \mathcal{P}+(1-p) \mathcal{Q})$ be the partition refining $\mathcal{W}$ in the following way: on $P$ we apply the (restriction of) partition $\mathcal{P}$, on $Q$ we apply the (restriction of) partition $\mathcal{Q}$.

In terms of symbolic dynamics, the $\mathcal{R}$-process can be imagined in as follows: each point $x \in \mathcal{X}$ has its (unilateral or bilateral, depending on the invertibility of $T$ ) $\mathcal{P}$-name, $\mathcal{Q}$-name, and $\mathcal{W}$-name. Then the $\mathcal{R}$-name of $x$ coincides with the $\mathcal{P}$-name along these coordinates, where the $\mathcal{W}$-name reads " $P$ ", otherwise (i.e., where the $\mathcal{W}$-name reads " $Q$ ") it coincides with the $\mathcal{Q}$-name. The first kind occurs in blocks of length precisely $\lfloor p n\rfloor$, alternated by blocks of the second kind, each having length at least $n-\lfloor p n\rfloor$ (and, typically, not much longer).

Lemma 2.4 Let $h_{1}$ and $h_{2}$ denote the entropies of the $\mathcal{P}$-process and $\mathcal{Q}$-process, respectively. Fix a coefficient $0<p<1$ and any $\delta>0$. Then, for $n$ large enough (regardless of the particular choice of an $n$-tower $)$, the entropy of the process generated by $\operatorname{MIX}_{n}((p \mathcal{P}+(1-$ p) $\mathcal{Q})$ does not exceed $p h_{1}+(1-p) h_{2}+\delta$.

Proof The mixed process in the symbolic form corresponds to an invariant measure on $\Lambda^{\mathbb{S}}$, where $\Lambda=\Lambda_{1} \cup \Lambda_{2}, \Lambda_{1}$ is a labeling of $\mathcal{P}, \Lambda_{2}$ is a labeling of $\mathcal{Q}$, and $\mathbb{S}=\mathbb{Z}$ or $\mathbb{N}_{0}$. It is rather obvious, that with $n \rightarrow \infty$, this measure approaches (in the weak-star topology) the convex combination $p \mu_{1}+(1-p) \mu_{2}$, where $\mu_{1}$ and $\mu_{2}$ are the invariant measures corresponding to the symbolic representations of the processes generated by $\mathcal{P}$ and $\mathcal{Q}$, respectively. Now the assertion follows from affinity and upper semicontinuity of the entropy function on invariant measures in symbolic systems.

1 The idea of the proof was suggested by Benjamin Weiss. 
Remark 2.5 More is true: the entropy of the process generated by $\operatorname{MIX}_{n}(p \mathcal{P}+(1-p) \mathcal{Q})$ converges (with $n$ ) to $p h_{1}+(1-p) h_{2}$, but this fact will not be used (and its proof requires more tools).

Proof of Theorem 2.2 By the Sinai Theorem, the system $(\mathcal{X}, \mathcal{F}, \mu, T)$ has the Bernoulli shift with entropy $c$ (which, by the Ornstein Theorem, is unique) as a measure-theoretic factor. Using a theorem of Weiss [13, Theorem 3.14], we can find a strictly ergodic model $\left(X^{\prime}, T^{\prime}\right)$ of $(\mathcal{X}, \mathcal{F}, \mu, T)$ which has the selected strictly ergodic model $\left(X_{1}, S_{1}\right)$ of the Bernoulli shift as a topological factor. Moreover, $\left(X^{\prime}, T^{\prime}\right)$ is zero-dimensional. Let $\mathcal{P}_{1}$ be the partition of $X^{\prime}$ by the cylinder sets corresponding to single symbols of the alphabet used in $\left(X_{1}, S_{1}\right)$. Next, let $\mathcal{P}_{2}, \mathcal{P}_{3}, \ldots$ be a sequence of clopen partitions of $X^{\prime}$ such that $\mathcal{P}_{k+1} \succcurlyeq \mathcal{P}_{k}$ for each $k \geq 1$ and $\operatorname{diam}\left(\mathcal{P}_{k}\right) \rightarrow 0$. For each $k$, the $\mathcal{P}_{k}$-process is a symbolic topological factor of $\left(X^{\prime}, T^{\prime}\right)$; the first one is the Bernoulli model, the $(k+1)$ st process factors topologically onto the $k$ th one, and the inverse limit equals $\left(X^{\prime}, T^{\prime}\right)$. If $h<C$, all these processes have entropies strictly smaller than $C$ and the proof is finished at this point.

The nontrivial part of the proof concerns the case $h=C$. In order to unify the construction, if additionally $c=0$ (and $\mathcal{P}_{1}$ does not generate a nontrivial Bernoulli system), we temporarily shift the enumeration by letting $\mathcal{P}_{0}$ be the trivial partition, while for $\mathcal{P}_{1}$ we choose a clopen partition which generates a process (necessarily strictly ergodic) with positive entropy $c^{\prime}<$ $C$ (clearly, such a partition exists). At the end of the construction we will shift back the enumeration, to fit it to the formulation of the theorem.

We are going to create a sequence of clopen partitions $\mathcal{R}_{k}$ of $X^{\prime}$, leading to the desired sequence of symbolic factors $\left(X_{k}, S_{k}\right)$. We begin by letting $\mathcal{R}_{1}=\mathcal{P}_{1}$.

Let $p_{1}, p_{2}, \ldots$ be a sequence of coefficients (strictly between 0 and 1), increasing to 1 . We will be using Rokhlin $n$-towers as in Lemma 2.4, but they need to satisfy two additional requirements:

(1) the towers must be measurable with respect to the factor generated by $\mathcal{R}_{1}$,

(2) the levels of the towers (and hence the reminder) must be clopen.

The first condition can be easily satisfied by simply choosing the towers within the factor system $\left(X_{1}, S_{1}\right)$ (this is the reason why we could not accept this system to be trivial) and then lifting them to $\left(X^{\prime}, T^{\prime}\right)$. The second condition can be obtained by a standard three-step approximation, using regularity (we slightly shrink the levels to make them closed), continuity (we slightly enlarge the levels to make them open) and zero-dimensionality (we shrink the levels again to make them clopen). Of course, these modifications must be performed within the factor $\left(X_{1}, S_{1}\right)$, before lifting to $\left(X^{\prime}, T^{\prime}\right)$. The $\mathcal{R}_{1}$-process (equal to $\left.\left(X_{1}, S_{1}\right)\right)$ has entropy $c$ (or $c^{\prime}$ ) strictly smaller than $C$.

Step $k+1$ Suppose we have defined a clopen partition $\mathcal{R}_{k}$ so that the $\mathcal{R}_{k}$-process $\left(X_{k}, S_{k}\right)$ has entropy strictly smaller than $C$. Because the weighted average with coefficients $p_{k+1}$ and $\left(1-p_{k+1}\right)$ of the entropies of the $\mathcal{P}_{k+1}$-process and the $\mathcal{R}_{k}$-process is strictly smaller than $C$, using Lemma 2.4, we can create their mix $\mathcal{R}_{k+1}=\operatorname{MIX}_{n_{k+1}}\left(p_{k+1} \mathcal{P}_{k+1}+\left(1-p_{k+1}\right) \mathcal{R}_{k}\right)$, with $n_{k+1}$ large enough so that the mixed process has entropy strictly smaller than $C$.

It is obvious that since for large $k$ the coefficient $p_{k+1}$ is close to 1 , the partition $\mathcal{R}_{k+1}$ is a good approximation of $\mathcal{P}_{k+1}$ (in the sense of measure). This implies that the inverse limit $(X, T)$ of the $\mathcal{R}_{k}$-processes is measure-theoretically isomorphic to the inverse limit of the $\mathcal{P}_{k}$-processes, while the latter equals $\left(X^{\prime}, T^{\prime}\right)$ and is a model for $(\mathcal{X}, \mathcal{F}, \mu, T)$. The only remaining difficulty is to verify that the $\mathcal{R}_{k}$-process is a topological factor of the $\mathcal{R}_{k+1^{-}}$process, for each $k \geq 1$.

If we agree that each partition $\mathcal{P}_{k}$ is labeled by an alphabet $\Lambda_{k}$ (these alphabets being pairwise disjoint), then the $\mathcal{R}_{k}$-names contain symbols from all alphabets $\Lambda_{1}$ through $\Lambda_{k}$. In 
the $(k+1)$ st step some of these symbols are replaced by the "refined" symbols from $\Lambda_{k+1}$ (recall that $\mathcal{P}_{k+1}$ is finer than each of $\mathcal{P}_{1}$ through $\mathcal{P}_{k}$ ). In order to reconstruct the $\mathcal{R}_{k}$-name (of some point $x$ ) from its $\mathcal{R}_{k+1}$-name, we need to find all symbols from $\Lambda_{k+1}$ and replace them by the corresponding "cruder" symbols from the alphabets $\Lambda_{1}$ through $\Lambda_{k}$. Here, at every such place (say, at coordinate $i$ in the name of $x$ ) we need to decide which of these $k$ alphabets should be used. For that we need to know the position of $T^{i} x$ with respect to all the towers used in steps $1, \ldots, k$. This can be determined as follows: at first we replace all symbols in the $\mathcal{R}_{k+1}$-name by the "crudest" symbols from $\Lambda_{1}$. In this manner we reconstruct the $\mathcal{R}_{1}$-name of $x$. Now, since all the towers are clopen and measurable with respect to the $\mathcal{R}_{1}$-process, the position of $T^{i} x$ with respect to these towers can be decided in a continuous (i.e., with finite coding horizon) procedure. The proof is now complete.

Remark 2.6 We believe that Theorem 2.2 holds also for noninvertible systems $(\mathcal{X}, \mathcal{F}, \mu, T)$ with $\left(X_{1}, S_{1}\right)$ being a strictly ergodic model of a one-sided Bernoulli shift with entropy $c$. There are, however, three problems, of which only one we know how to resolve. Of course, none of them appears in case $c=0$, and Theorem 2.2 holds in this case also for endomorphisms, with $\left(X_{1}, S_{1}\right)$ being the trivial unilateral subshift over one symbol. The three problems are:

(1) Sinai's theorem for endomorphisms $(\mathcal{X}, \mathcal{F}, \mu, T)$ does hold (the original proof works) and it produces some unilateral Bernoulli shift with entropy $c$ as a factor. However, since the Ornstein Theorem is known to fail for unilateral Bernoulli shifts, the above factor may depend on $(\mathcal{X}, \mathcal{F}, \mu, T)$. If $c>\log 2$ then all Bernoulli shifts with entropy $c$ are weakly isomorphic (each of them requires at least 3 symbols and a theorem of del Junco [4, Theorem 1] applies), which enables us to pick any one of them to create $\left(X_{1}, S_{1}\right)$. But if $c \leq \log 2$, it may happen that for endomorphisms in some subclass (of the class with entropies in $I$ ) the Bernoulli factor with entropy $c$ requires two symbols, while in the remaining subclass-three or more. It is unknown whether there is a factor relation (in any direction) between these two kinds of Bernoulli shifts, so we cannot choose one Bernoulli shift good (as a factor) for the entire class. We solve this problem by creating two "quasi universal" systems: one based on the Bernoulli shift on two symbols (it is unique, for fixed entropy), and another, based on a Bernoulli shift on three symbols (these are weakly isomorphic, so any of them can serve). In the end, the union of the two "quasi universal" systems will be universal.

(2) An analog of the Jewett-Krieger Theorem for noninvertible systems has been proved by Rosenthal ([9], the same result follows also from [2]), but it cannot be hoped that, in case of finite entropy, the strictly ergodic model can always be chosen a unilateral subshift. Such a subshift obviously admits a finite forward generator, while not every endomorphism does. On the other hand, any unilateral Bernoulli shift by definition admits a finite forward generator, so one can hope that a strictly ergodic unilateral subshift model $\left(X_{1}, S_{1}\right)$ exists. But this is not clear at all.

(3) We have not been able to find a noninvertible version of Weiss' theorem, which is needed to define $\left(X^{\prime}, T^{\prime}\right)$. We strongly believe that the necessary modification is possible, but the task requires extensive work, not fitting in the frames of this exposition.

\section{Universal Systems for Closed Entropy Intervals}

Theorem 3.1 Let $I=[c, C]$, where $0 \leq c<C \leq \infty$. Then there exists a universal system for the class $\mathcal{H}_{I}^{\mathrm{a} a}$. If $c=0$, a universal system for the class $\mathcal{H}_{I}^{\mathrm{e}}$ also exists. 
Proof We fix a strictly ergodic subshift $\left(X_{1}, S_{1}\right)$ which is a model of the Bernoulli shift with entropy $c$ (if $c=0$ this is a trivial subshift over a one-element alphabet) and we let $\Lambda_{1}$ denote the alphabet used in $X_{1}$. For $l \geq 2$ let $\Lambda_{l}=\{0,1, \ldots, l-1\}$. We define $Y=X_{1} \times \prod_{l>2} \Lambda_{l}^{\mathbb{S}}$, where $\mathbb{S}=\mathbb{Z}$ or $\mathbb{N}_{0}$, depending on invertibility (recall that the noninvertible case is handled only for trivial $\left.\left(X_{1}, S_{1}\right)\right)$. So defined $Y$ is a collection of matrices $y=[y(l, n)]_{l \geq 1, n \in \mathbb{S}}$ consisting of countably many rows, where the $l$ th row $y_{l}$ is an infinite (bilateral or unilateral) sequence over $\Lambda_{l}$. We act on elements $y$ by the left shift $T$, i.e. $T y=y^{\prime}$ where $y^{\prime}(l, n)=$ $y(l, n+1)$ for all $l, n$.

An $l$-rectangle is a finite matrix of size $l \times 2^{l}$, such that in row $i$ it contains elements of $\Lambda_{i}(i=1,2, \ldots, l)$. We say that an $l$-rectangle $R$ occurs in $y \in Y$ if $R=[y(i, j): i=$ $\left.1,2, \ldots, l, j=n, n+1, \ldots, n+2^{l}-1\right]$ for some $n \in \mathbb{S}$. If $B$ is a block of length $2^{l}$ over the alphabet $\Lambda_{1}$ (we will write $B \in \Lambda_{1}^{2^{l}}$ ), then by $\operatorname{Rec}_{l, B}(y)$ we denote the collection of all $l$-rectangles occurring in $y$ and having the block $B$ in the first row (if $B$ does not occur in $X_{1}$, this collection is empty for all $y \in Y$ ).

We define $Z$ as a subset of $Y$ consisting of elements $y$ satisfying, for all $l \geq 2$ and any $B \in \Lambda_{1}^{2^{l}}$, the inequality

$$
\# \operatorname{Rec}_{l, B}(y) \leq \exp \left(2^{l}(C-c)\right) .
$$

It is obvious that $Z$ is a closed and $T$-invariant subset of $Y$. Let $F$ denote the restriction of $T$ to $Z$. We will show that $(Z, F)$ is a universal system in question.

For $l \geq 1$, we denote by $\left(Z_{l}, F_{l}\right)$ the topological (symbolic) factor of $(Z, F)$ obtained by the restriction to the first $l$ rows (in particular, $\left(Z_{1}, F_{1}\right)=\left(X_{1}, S_{1}\right)$ ). Let $\mathcal{Q}_{l}$ denote the clopen partition of $Z$ (and, in fact of $Z_{l}$ ) defined by fixing the entries $y(1,0), y(2,0), \ldots, y(l, 0)$. Then $\left\{\mathcal{Q}_{l}\right\}$ is a refining sequence of partitions in $Z$ and the $\mathcal{Q}_{l}$-process coincides with $\left(Z_{l}, F_{l}\right)$. Note that $\bigvee_{n=0}^{2^{l}-1} F_{l}^{-n} \mathcal{Q}_{l}$ partitions $Z$ into cylinder sets corresponding precisely to the $l$ rectangles. Consider an ergodic measure $v$ supported by $Z$. Let $y \in Z$ be a point generic for $v$ and let $v_{l}$ denote the image of $v$ by projection onto $Z_{l}$. Then by well known facts on entropy and by (3.1), for any $l \geq 2$ we have

$$
h_{\nu_{l}}\left(F_{l} \mid X_{1}\right) \leq \frac{1}{2^{l}} H_{\nu_{l}}\left(\bigvee_{n=0}^{2^{l}-1} F_{l}^{-n} \mathcal{Q}_{l} \mid \bigvee_{n=0}^{2^{l}-1} F_{1}^{-n} \mathcal{Q}_{1}\right) \leq \frac{1}{2^{l}} \sup _{B \in \Lambda_{1}^{2^{l}}} \log \left(\# \operatorname{Rec}_{l, B}(y)\right) \leq C-c .
$$

The entropy of $v$, which equals

$$
h_{v}(F)=\sup _{l} h_{v_{l}}\left(F_{l}\right)=\sup _{l} h_{v_{l}}\left(F_{l} \mid X_{1}\right)+h_{v_{1}}\left(F_{1}\right)
$$

is hence contained between $c$ and $C$. In this way, $Z$ satisfies the second postulate for being universal in the class $\mathcal{H}_{I}^{\mathrm{a} a}$ (or $\mathcal{H}_{I}^{\mathrm{e}}$ ).

It remains to show that every ergodic measure-theoretic system $(\mathcal{X}, \mathcal{F}, \mu, T)$ satisfying $h_{\mu}(T) \in I$ is isomorphic to an ergodic $F$-invariant measure $v$ supported by $Z$. By Theorem 2.2 , there exists a strictly ergodic zero-dimensional model $(X, T)$ of $(\mathcal{X}, \mathcal{F}, \mu, T)$, which is an inverse limit of uniquely ergodic symbolic factors $\left(X_{k}, T_{k}\right)$, each of entropy strictly smaller than $C$ and with $\left(X_{1}, S_{1}\right)$ equal to the system appearing as the first row factor of $(Z, F)$. Let $\mathcal{R}_{k}$ be a clopen partition of $X$ which generates the factor $\left(X_{k}, T_{k}\right)$. Replacing, if necessary $\mathcal{R}_{k}$ by $\bigvee_{i=1}^{k} \mathcal{R}_{i}$, we can assume that $\mathcal{R}_{k+1} \succcurlyeq \mathcal{R}_{k}$ for every $k \geq 1$. Let $r_{k}$ be the cardinality of $\mathcal{R}_{k}$. Of course, we can injectively label $\mathcal{R}_{k}$ with the alphabet $\Lambda_{l}$ (perhaps not using all symbols) for any $l \geq r_{k}$. Since $h_{\text {top }}\left(T_{k}\right)<C$, applying the variational principle for topological entropy (see [3, Theorem 6.8.8]) and strict ergodicity, we get $h_{\text {top }}\left(T_{k} \mid X_{1}\right)<C-c$. Next, by subadditivity of topological conditional entropy for open covers, there exists a positive 
integer $m_{k}$ such that

$$
\frac{1}{2^{m}} H_{\text {top }}\left(\bigvee_{n=0}^{2^{m}-1} T_{k}^{-n} \mathcal{R}_{k} \mid \bigvee_{n=0}^{2^{m}-1} T_{1}^{-n} \mathcal{R}_{1}\right) \leq C-c
$$

for any $m \geq m_{k}$.

Let $l_{1}=1$ and then put inductively $l_{k+1}=\max \left\{r_{k+1}, m_{k+1}, l_{k}+1\right\}$ for all $k \geq 1$. In this manner we guarantee three properties:

- the sequence $\left\{l_{k}\right\}_{k \geq 1}$ starts with 1 and strictly increases,

- for each $k \geq 1$ the partition $\mathcal{R}_{k}$ can be labeled using the alphabet $\Lambda_{l_{k}}$,

- we have the inequality (3.2) for $l_{k}$ in place of $m$.

Now we can produce a special, topologically conjugate, array representation of $(X, T)$, by placing each symbolic factor $\left(X_{k}, T_{k}\right)$ in row number $l_{k}$ (instead of in row $k$ ) and filling all other (unused) rows by zeros. From now on, $(X, T)$ will denote this representation. We will argue that so understood $(X, T)$ is a subsystem of $(Z, F)$. Indeed, the alphabet used in the $l$ th row of $(X, T)$ is contained in $\Lambda_{l}$, for every $l \geq 1$. Further, notice that since $\mathcal{R}_{k}$ refines all $\mathcal{R}_{i}$ with $i \leq k$ and the added rows are filled with zeros, we can easily estimate the cardinality of $l$-rectangles appearing in $X$ with a fixed block $B$ in the first row (this will of course estimate the number of such $l$-rectangles appearing in each individual element of $X$ ), as follows.

If $l=l_{k}$ for some $k \geq 2$, this cardinality equals that of

$$
\left.\left(\bigvee_{n=0}^{2^{l} k-1} T_{k}^{-n} \mathcal{R}_{k}\right)\right|_{B}
$$

hence does not exceed $\exp \left(2^{l_{k}}(C-c)\right)$, as required.

For $l$ not of the form $l_{k}$, we let $k$ be the largest index with $l_{k}<l$. Since all rows numbered $l_{k}+1, l_{k}+2, \ldots, l$ are filled with zeros, the cardinality of $l$-rectangles with a fixed first row $B$ equals that of

$$
\left.\left(\bigvee_{n=0}^{2^{l}-1} T_{k}^{-n} \mathcal{R}_{k}\right)\right|_{B}
$$

which, by submultiplicativity, does not exceed

$$
\left.\prod_{j=1}^{2^{l-l_{k}}} \#\left(\bigvee_{n=0}^{2^{l_{k}}-1} T_{k}^{-n} \mathcal{R}_{k}\right)\right|_{B_{j}}
$$

where $B=B_{1} B_{2} \ldots B_{2^{l-l_{k}}}$ is the partition of $B$ into $2^{l-l_{k}}$ blocks of equal lengths $2^{l_{k}}$. Since, by (3.2) (applied to $m=l_{k}$ ), each of the multipliers does not exceed $\exp \left(2^{l_{k}}(C-c)\right)$, the total cardinality in question is bounded by $\exp \left(2^{l}(C-c)\right)$. We have proved that $X$ is contained in $Z$, hence the (unique) ergodic measure (call it $v$ ) supported by $X$ is in fact supported by $Z$, and it is isomorphic to the initially selected measure $\mu$. This ends the proof of the first postulate of universality of $(Z, F)$, and hence the entire proof. 


\section{Universal Systems for Open Entropy Intervals}

Theorem 4.1 If $I=[0, C)$ or $(c, C)$ (with $0 \leq c<C \leq \infty)$ there exists a universal system for the class $\mathcal{H}_{I}^{\mathrm{a} a}$. If $I=[0, C)$ or $(c, \infty)$ then also $\mathcal{H}_{I}^{\mathrm{e}}$ admits a universal system.

Proof We begin by constructing universal systems for $\mathcal{H}_{[0, C)}^{\mathrm{a} a}$ and $\mathcal{H}_{[0, C)}^{\mathrm{e}}$. This is very easy. We take universal systems for $\mathcal{H}_{\left[0, C-\frac{1}{n}\right]}^{\mathrm{a} a}$ (with $n>\frac{1}{C}$ ) in case $C<\infty$, or just $\mathcal{H}_{[0, n]}^{\mathrm{a} a}$ in case $C=\infty$ obtained in Theorem 3.1, and then arrange (in some larger space) a disjoint union over increasing $n$ of copies of such systems shrinking in diameter and accumulating at a fixpoint. The same proof works in the noninvertible case.

We pass to $\mathcal{H}_{(c, C)}^{\mathrm{a} a}$. First, we will need a special topological (invertible) system $\left(X_{1}, T_{1}\right)$. It should have countably many ergodic measures $v_{n}$. We need all these measures to be Bernoulli with entropies smaller than $\frac{c+C}{2}$. The entropies of these measures should strictly decrease in $n$ and converge to $c$, while the measures themselves should converge (in the weak-star topology) to $v_{1}$ (which has the largest entropy in the sequence). The existence of such a system (on the Cantor set) follows from [5], as well as from [2, Corollary 5.2] (also in the noninvertible case).

Next, we let $\left(X_{2}, T_{2}\right)$ be the universal system for $\mathcal{H}_{\left[0, C-h\left(v_{1}\right)\right)}^{\mathrm{a} a}$ and we define $(Z, F)$ as the direct product $\left(X_{1} \times X_{2}, T_{1} \times T_{2}\right)$. We will argue that this is the desired universal system.

Clearly, any invariant measure on $(Z, F)$ has entropy strictly between $c$ and $C$. On the other hand, any system $(\mathcal{X}, \mathcal{F}, \mu, T)$ with entropy $h$ in this range has a (measure-theoretic) Bernoulli factor isomorphic to some $v_{n}$ (we choose $n$ to be the smallest index with $h>h\left(v_{n}\right)$ ) and then it is a joining of $v_{n}$ with an automorphism of entropy $h-h\left(v_{n}\right)+\epsilon$, where $\epsilon$ is arbitrarily small ( $\epsilon$-independent completion; see e.g. [3, Corollary 4.4.8]). If $n=1$ then $h-h\left(v_{1}\right)+\epsilon$ can be made smaller than $C-h\left(v_{1}\right)$, and thus the $\epsilon$-independent completion is represented by an invariant measure $v^{\prime}$ on $X_{2}$. The product system $(Z, F)$ supports all joinings of $v_{1}$ with $v^{\prime}$, in particular the one isomorphic to $\mu$. If $n>1$ then the difference $h-h\left(v_{n}\right)$ does not exceed $h\left(v_{1}\right)-c$, which is smaller than $C-h\left(v_{1}\right)$, so $h-h\left(v_{n}\right)+\epsilon$ can be made smaller than $C-h\left(v_{1}\right)$ and the rest of the argument applies just as for $n=$ 1.

In case of endomorphisms, the systems $\left(X_{1}, T_{1}\right)$ and $\left(X_{2}, T_{2}\right)$ can be constructed, using the same tools, with the measures $v_{n}$ representing unilateral Bernoulli shifts. It is true that if an endomorphism $(\mathcal{X}, \mathcal{F}, \mu, T)$ has entropy $h \in I$ then it has one of the Bernoulli measures $v_{n}$ as a measure-theoretic factor (the inequality between $h$ and entropy of $v_{n}$ is sharp hence we can apply Sinai Theorem combined with [4, Theorem 2]). The weak spot is the existence of an $\epsilon$-independent completion. It is known that [3, Corollary 4.4.8] fails for endomorphisms (see [3, Remark 4.4.9]). Thus, we can safely conduct the proof only if $C=\infty$ because then on $\left(X_{2}, T_{2}\right)$ we can find a measure $v^{\prime}$ isomorphic to $\mu$ and on $(Z, F)$ use the "factor joining" supported by the graph of the measure-theoretic factor map from $v^{\prime}$ to $v_{n}$, which is also isomorphic to $\mu$.

\section{All Nondegenerate Intervals}

Corollary 5.1 The following classes admit universal systems: for automorphisms: $\mathcal{H}_{I}^{\mathrm{a} a}$ for any nondegenerate interval $I \subset[0, \infty]$. For endomorphisms: $\mathcal{H}_{[0, C]}^{\mathrm{e}}$ and $\mathcal{H}_{[0, C)}^{\mathrm{e}}$ (with $0<$ $C \leq \infty), \mathcal{H}_{(c, \infty)}^{\mathrm{e}}$, and $\mathcal{H}_{(c, \infty]}^{\mathrm{e}}$ (with $\left.0 \leq c<\infty\right)$. 
Proof For automorphisms: any nondegenerate interval is a union of at most one nondegenerate closed and one open interval. Thus a disjoint union of at most two universal systems obtained in Theorems 3.1 and 4.1 will serve.

For endomorphisms: the first three cases are included in Theorems 3.1 and 4.1, the last one, with $I=(c, \infty)$, is obtained by the same proof as for $I=(c, \infty)$ with $\left(X_{2}, T_{2}\right)$ being the universal system for the class $H_{[0, \infty]}^{\mathrm{e}}$ of all endomorphisms.

\section{Minimal Universal Models for Classes of Aperiodic Systems}

In this section we address classes consisting of aperiodic ergodic systems. Clearly if $0 \notin I$ then the classes $\mathcal{H}_{I}^{\mathrm{a} a}$ and $\mathcal{H}_{I}^{\mathrm{e}}$ contain only aperiodic systems. If $0 \in I$, we have new classes $\tilde{\mathcal{H}}_{I}^{\mathrm{a} a}$ and $\tilde{\mathcal{H}}_{I}^{\mathrm{e}}$ obtained by discarding periodic measures from $\mathcal{H}_{I}^{\mathrm{a} a}$ and $\mathcal{H}_{I}^{\mathrm{e}}$. Using a special tool (markers), and the minimal models theorem, we can prove not only the existence of universal systems for these new classes, but also we can make these systems minimal.

\section{Theorem 6.1 The following classes admit minimal universal systems:}

- if $0 \notin I$, any class $\mathcal{H}_{I}^{\mathrm{a} a}$ or $\mathcal{H}_{I}^{\mathrm{e}}$ such that the universal system for this class exists (e.g. as specified in Corollary 5.1),

- if $0 \in I$, both $\tilde{\mathcal{H}}_{I}^{\mathrm{a} a}$ and $\tilde{\mathcal{H}}_{I}^{\mathrm{e}}$.

Proof We begin by showing that in case $0 \in I$, the classes $\tilde{\mathcal{H}}_{I}^{\mathrm{a} a}$ and $\tilde{\mathcal{H}}_{I}^{\mathrm{e}}$ admit universal systems. Let $(Z, F)$ be the universal system for the class with the "tilde" removed (such a system exists, by Corollary 5.1). By [2, Lemma 2.4], for each $n$ there exists a set $G_{n}$ visited by every orbit with gaps ranging between $n$ and $2 n+1$ and such that its boundary is contained in the set of points which are periodic or asymptotic to periodic. We will only use a subsequence $G_{n_{k}}$ for indices $n_{k}$ growing so fast that the series of the binary entropies

$$
\sum_{k=1}^{\infty} H\left(\frac{1}{n_{k}}, 1-\frac{1}{n_{k}}\right)
$$

converges to a number strictly smaller than $C$ (recall that $I=[0, C)$ or $[0, C]$ for some $0<C \leq \infty)$. Since $(Z, F)$ is zero-dimensional, we can use its array representation and mark the visits of every orbit in $G_{n_{k}}$ in row number $k$. We do it by placing some markers, say stars, next to the symbols at coordinates corresponding to the times of the visits. We apply this only for arrays which are not asymptotic to periodic and then we let $(\tilde{Z}, \tilde{S})$ be the closure of the collection of so obtained arrays with markers. It is clear that by removing the stars, $(\tilde{Z}, \tilde{F})$ factors onto a subsystem $\left(Z^{\prime}, F^{\prime}\right)$ of $(Z, F)$, which supports all its aperiodic ergodic measures and perhaps contains some periodic orbits as well (it can be proved that $(\tilde{Z}, \tilde{F})$ factors onto $(Z, F)$, but this will not be necessary). Because the boundaries of the sets $G_{n_{k}}$ have measure zero for every aperiodic measure, the factor map is invertible modulo each such measure. Thus $(\tilde{Z}, \tilde{F})$ fulfills the first requirement for being universal for the class with the "tilde". In order to verify the second postulate, we only need to check the preimages of periodic measures (whether they are aperiodic and with entropy smaller than $C$ ). It is so, because every periodic point in $Z^{\prime}$ lifts to an array with markers distributed in row $k$ with gaps ranging between $n_{k}$ and $2 n_{k}+1$. Every such array is obviously aperiodic, and the entropy on its orbit closure is generated only by the processes of markers (the original symbols in each row are periodic). But the lower bound on the gaps between the markers in row $k$ implies that the markers in this row can generate entropy at most $H\left(\frac{1}{n_{k}}, 1-\frac{1}{n_{k}}\right)$, hence (summing over 
all rows) the marker processes have the joint entropy strictly smaller than $C$, as required. We have shown that $(\tilde{Z}, \tilde{F})$ is universal for the class in question.

Once this is established, we can pass to creating minimal universal models. This is immediate (modulo appropriate tools). The universal system, $(Z, F)$ or $(\tilde{Z}, \tilde{F})$, for a class consisting of aperiodic measures (as listed in the formulation of the theorem) is zero-dimensional and obviously aperiodic. Then [1, Theorem 1] provides a zero-dimensional minimal system with exactly the same simplex of invariant measures. Clearly, this minimal system is universal for the same class.

Remark 6.2 The above theorem strengthens the result from [11] in two ways: it applies to endomorphism as well as automorphisms, and produces universal systems in our stronger understanding. In one aspect it is weaker: our universal system is not a subshift.

\section{Open Questions}

Let us gather the unsolved cases. We begin with the one for which we strongly believe that the answer is positive:

Question 7.1 Do there exist universal systems for the classes $\mathcal{H}_{I}^{\mathrm{e}}$, where I is bounded, nondegenerate, and does not contain 0 ?

For the next question we are inclined toward the negative answer (by similarity to the case with $c=0$ ), but not enough to conjecture:

Question 7.2 Do there exist universal systems for the classes $\mathcal{H}_{\{c\}}^{\mathrm{a} a}$ and $\mathcal{H}_{\{c\}}^{\mathrm{e}}$, where $c>0$ ?

The last question is of different nature and perhaps it is the most interesting:

Question 7.3 Does there exist a bilateral subshift universal for $\mathcal{H}_{[0, C]}^{\mathrm{a} a}$ or a unilateral subshift universal for $\mathcal{H}_{[0, C]}^{\mathrm{e}}$ (with $\left.C<\infty\right)$ ? The same question can be asked for intervals $[c, C]$ or $(c, C]$ with $c<C<\infty$.

The type of intervals (the upper end finite and closed) does not immediately exclude such a possibility but our methods inevitably lead to nonexpansive zero-dimensional systems. If the answer was positive then we could apply the "faces technique" of [2] to answer positively Question 7.2.

Acknowledgements The research of both authors is supported by the NCN (National Science Center, Poland) Grant 2013/08/A/ST1/00275.

Open Access This article is distributed under the terms of the Creative Commons Attribution 4.0 International License (http://creativecommons.org/licenses/by/4.0/), which permits unrestricted use, distribution, and reproduction in any medium, provided you give appropriate credit to the original author(s) and the source, provide a link to the Creative Commons license, and indicate if changes were made.

\section{References}

1. Downarowicz, T.: Minimal models for noninvertible and not uniquely ergodic systems. Isr. J. Math. 156, 93-110 (2006)

2. Downarowicz, T.: Faces of simplexes of invariant measures. Isr. J. Math. 165, 189-210 (2008) 
3. Downarowicz, T.: Entropy in Dynamical Systems. New Mathematical Monographs, vol. 18. Cambridge University Press, Cambridge (2011)

4. del Junco, A.: Finitary codes between one-sided Bernoulli shifts. Ergod. Theory Dyn. Syst. 1, 285-301 (1981)

5. Kornfeld, I., Ormes, N.: Topological realizations of families of ergodic automorphisms, multitowers and orbit equivalence. Isr. J. Math. 155, 335-357 (2006)

6. Krieger, W.: On unique ergodicity. In: Proceedings of 6th Berkeley Symposium on Mathematical Statistics and Probability, pp. 327-346 (1970)

7. Lind, D.A., Thouvenot, J.-P.: Measure-preserving homeomorphisms of the torus represent all finite entropy ergodic transformations. Math. Syst. Theory 11, 275-282 (1977)

8. Quas, A., Soo, T.: Ergodic universality of some topological dynamical systems. Preprint arXiv:1208.3501

9. Rosenthal, A.: Strictly ergodic models for non-invertible transformations. Isr. J. Math. 64, 57-72 (1988)

10. Serafin, J.: Non-existence of a universal zero-entropy system. Isr. J. Math. 194, 349-358 (2013)

11. Shilon, O., Weiss, B.: Universal minimal topological dynamical system. Isr. J. Math. 160, 119-141 (2007)

12. Weiss, B.: Countable generators in dynamics-universal minimal models. Contemp. Math. 94, 321-326 (1989)

13. Weiss, B.: Single orbit dynamics. CBMS Regional Conference Series in Mathematics (2000) 